\title{
Scale-Free Properties of Board and Director Networks Quantities
}

\author{
D. SIUDAK ${ }^{a, *}$ AND A. SANKOWSKA ${ }^{b}$ \\ ${ }^{a}$ Lodz University of Technology, Department of Organisation and Administration, \\ Piotrkowska 266, 90-924 Lodz, Poland \\ ${ }^{b}$ Warsaw University of Technology, Warsaw, Poland \\ (Received April 24, 2016; revised version August 18, 2016; in final form October 16, 2016)
}

\begin{abstract}
It is often claimed that corporate and board networks display a scale-free nature, but there is no robust support for this nature. Based on data concerning the corporate board and director networks from companies listed on the Warsaw Stock Exchange Market, we applied a rigorous approach to determine whether quantities of these networks, such as degree, board size, and directorship number, exhibit power-law distribution.
\end{abstract}

DOI: 10.12693/APhysPolA.130.1261

PACS/topics: $02.50 . \mathrm{Ng}, 05.90 .+\mathrm{m}$

\section{Introduction}

Networks are omnipresent in the current world $[1,2]$. Presumably, the complexity in networks derived from the interconnectedness of vertices produces, through emergent behaviors, highly right-skewed histograms that are called scale-free [3]. Despite their significant variations in individual constituents, social, information, technological and biological networks are inclined to follow a universal law of power-law distribution [4-6]. For instance, it has been reported that metabolic networks [7], the WorldWide Web [8, 9], Internet [10], scientific collaboration and publications citation network [11-13], stock market prices network $[14,15]$, stock ownership networks [16], movie actor network [17], network of sexual relationships [18], and inter-firm relationships network $[19,20]$ are scale-free.

An important consequence of the scale-free property is that several highly connected vertices (hubs) dominate the overall connectivity of a network [7]. The power-law degree distribution - the probability $P(k)$ of a vertex to have exactly $k$ degree (edges) - follows the function:

$$
P(k) \sim k^{-\alpha},
$$

where $\alpha>0$ is a constant parameter (scaling parameter, the exponent of the power-law distribution). $P(k)$ represents the histogram of the number of vertices with a given degree $k$ [21], where the fraction of vertices with degree $k$ is proportional to $1 / k^{\alpha}$. From a statistical point of view, large events in power-law distributions are more often expected than under the normal distribution [22].

As a standard, researchers rely on a scaling parameter value when drawing a conclusion regarding a scale-free property of networks. It is said that most real scale-free networks fall in a range $2<\alpha<3[3,23]$, which is used as a rule of thumb to determine whether graphs obey a power-law distribution. It is worth mentioning, though,

\footnotetext{
*corresponding author; e-mail: dariusz.siudak@p.lodz.pl
}

that there are exceptions. In the literature, there are reported, for instance, power-law graphs with exponents less than 2 or even with an exponent in $(2,+\infty)[24,25]$. Thus, in spite of this wide method usage, the sole estimation of the scaling parameter cannot guarantee that the analyzed data originates from a power-law distribution. The verifications of whether the degree distribution satisfies the power-law calls for more advanced method than either a visual form (graph) or the value of the exponent.

In particular, social networks, where interacting agents modify their strategies in response to the interactions of other agents, are adaptive systems that generate scalefree data. Out of social networks, it is the board and director networks that have attracted considerable interest [16, 26-28]. The board network reflects the network of boards connected through common directors, while the director network shows links between directors sharing a membership in the same board.

In particular, the occurrence of a skewed Pareto distribution of degree has been detected in board and director networks in some markets relying only on (1) simplified graphical methods, such as a visual inspection of raw data plot (histogram) $[26,27]$ or data plotted doubly on logarithmic scales which, according to the relation

$$
\ln P(x)=\ln C-\alpha \ln x
$$

should follow a straight line [22]; and (2) a value of the scaling parameter that is supposed to fall in the range between 2 and $3[3,23]$. These inaccurate methods suffering from severe biases can lead to false conclusions $[6,21,22,29,30]$ and cannot be trusted [22] because without a measure of goodness-of-fit, it is difficult to assess how well the data in question approximates a power-law distribution [29]. In these terms, the existing empirical evidence of power-laws degree distribution in board and directors networks $[26,28]$ is limited and questionable. Moreover, a more refined analysis of the corporate board network of companies listed on the Italian Stock market using chi-square statistics ruled out the scale-free nature of this type of network [27]. 
In addition to limited evidence of a power-law distribution of degree in board and director networks, the network distributions of other quantities have been largely ignored. The current paper fulfills this research gap and illuminates better the network properties of board and director networks. To this end, we analyze the distribution of several board and director network quantities, such as the board and director network degree, board size (number of directors in each single board), and directorship number (number of boards each single director serves). In this way, we aim to reconcile the inconsistent findings and expand the empirical evidence regarding the aforementioned network properties. The paper adds to several existing studies on the topic in the following ways: (1) by more strict assessment of director and board network scaling properties of degree distribution and (2) by analyzing other quantities of scale-free properties using different quantities than degree. In the paper, we adopted the methodology for detecting powerlaw distribution described in [31].

\section{Data set}

The examined data consisted of the board information of 902 companies listed on the main market at the Warsaw Stock Exchange (461 companies) and on the NewConnect market (441 firms) derived from December 2014. This data was obtained from Notoria database and checked for consistency. The corporate board network was constructed with 902 boards ( 518 for the largest component) as vertices connected by an edge if they share at least one director. The director network consisted of 5928 directors (3 282 for the largest component) as vertices connected if a given pair of directors serves on the same board. The fraction of the vertices in the largest component, $N_{L C} / N$, is $57.4 \%$ for board network and $55.4 \%$ for director network, respectively. The networks are undirected and unweighted.

\section{Results}

To assess the power-law properties, we adopted the procedure proposed by [31] developed for continuous and discrete-valued data. We estimated the power-law exponent and the lower bound $\left(x_{\min }\right)$ and used a goodnessof-fit test. The results of the goodness-of-fit tests for several quantities, namely, degree distributions in board and director networks, board size distribution, and directorship number distribution, are presented in Table I and Figs. 1-3.

If the resulting $p$-value that quantifies goodness-of-fit is greater than 0.1 for some lower bound $\left(x_{\min }\right)$, then the power-law distribution plausibly holds for the data above the cutoff $x_{\text {min }}$; otherwise, we rule out the power-law hypothesis, and the data are not likely to follow a powerlaw distribution. As seen in Table I, in all cases, except for degree distribution, in the entire director network, the power-law distribution can plausibly fit the data. Note

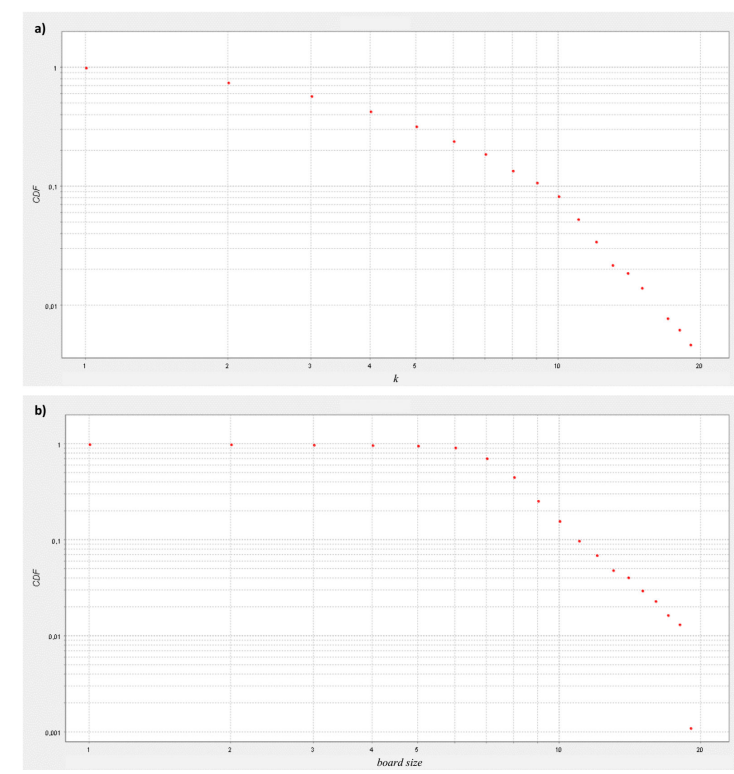

Fig. 1. The power-law distribution of board network quantities: (a) board - degree; (b) number of directors (board size). CDF - complementary cumulative distribution function.

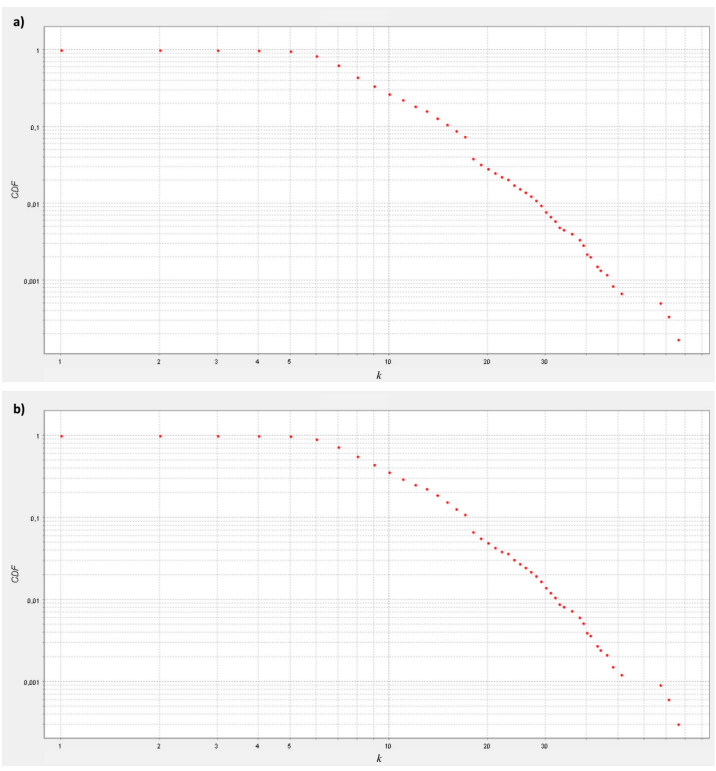

Fig. 2. The power-law distribution of director network quantities: (a) director - degree; (b) director - degree; the largest component.

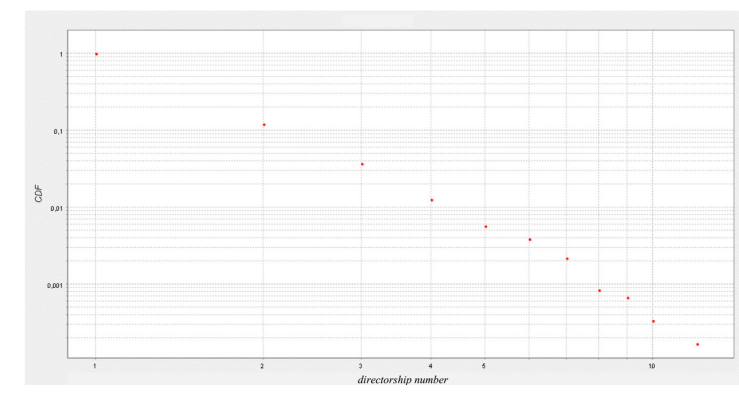

Fig. 3. The power-law distribution of number of boards per director (directorship number). 
that in the largest component of the director network, in contrast to the entire network, the degree distribution is likely to follow the power-law distribution (although for a very high lower bound $x_{\min }=27$ ).

TABLE I

Examination of power-law properties of network quantities.

\begin{tabular}{|c|c|c|c|c|c|c|c|c|}
\hline Network & Network quantity & $N$ & $k$ & $\begin{array}{c}\text { Lower } \\
\text { bound } \\
x_{\min }\end{array}$ & $\begin{array}{c}\text { The maximum } \\
\text { likelihood } \\
\text { estimator } \\
\text { of the } \\
\text { power-law } \\
\text { exponent } \alpha\end{array}$ & $p$-value & $\begin{array}{c}\text { Number of } \\
\text { iterations }\end{array}$ & $\begin{array}{l}\text { Support } \\
\text { for the } \\
\text { power-law } \\
\text { distribution }\end{array}$ \\
\hline board & degree & 902 & 2558 & 10 & 5.600 & 0.941 & 1000000 & plausible \\
\hline board & $\begin{array}{c}\text { number of directors } \\
\text { (board size) }\end{array}$ & & & 8 & 5.364 & 0.791 & 1000000 & plausible \\
\hline director & degree & 5928 & 52242 & 7 & 3.387 & 0.000 & 400000 & rejected \\
\hline director & degree; the largest component & 3282 & 32672 & 27 & 5.189 & 0.570 & 1000000 & plausible \\
\hline director & $\begin{array}{c}\text { number of boards } \\
\text { per director } \\
\text { (directorship number) }\end{array}$ & & & 4 & 3.961 & 0.560 & 1000000 & plausible \\
\hline
\end{tabular}

\section{Conclusions}

In this paper, using data on corporate board and director networks, we supported the power-law distribution in many quantities. This finding suggests that chairs in boards are not assigned at random in the sense of ErdösRenyi [32] because the degree distribution would likely follow the Poisson distribution instead.

Moreover, identifying that certain quantities plausibly follow a power-law distribution in empirical data suggests certain underlying generative mechanism for the given quantities. It has been discovered that scale-free networks are created through preferential attachment such that the probability of connecting to a vertex depends on the vertex degree $[3,17,31]$. This finding indicates that there are few boards (firms) and directors (persons) that, as centers of gravity, possess a greater capacity to attract others.

\section{Acknowledgments}

We acknowledge the support of the Polish National Science Centre, grant no. DEC-2013/11/B/HS4/00466.

\section{References}

[1] A.-L. Barabasi, E. Ravasz, T. Vicsek, Physica A 299 , 559 (2002).

[2] S.H. Strogatz, Nature 410, 268 (2001).

[3] R. Albert, A.-L. Barabasi, Rev. Mod. Phys. 74, 47 (2002).

[4] M.E.J. Newman, Networks: An Introduction, Oxford University Press, Oxford 2010.
[5] Z.-M. Lu, Y.-X. Su, S.-Z. Guo, Physica A 392, 3555 (2013).

[6] M.E.J. Newman, Contemp. Phys. 46, 323 (2005).

[7] H. Jeong, B. Tombor, R. Albert, Z.N. Oltvai, A.-L. Barabasi, Nature 407, 651 (2000).

[8] R. Albert, H. Jeong, A.-L. Barabási, Nature 401, 130 (1999).

[9] A.-L. Barabási, R. Albert, H. Jeong, Physica A 281, 69 (2000).

[10] M. Faloutsos, P. Faloutsos, C. Faloutsos, Comput. Commun. Rev. 29, 251 (1999).

[11] S. Redner, Europ. Phys. J. B 4, 131 (1998).

[12] A.L. Barabasi, H. Jeong, Z. Neda, E. Ravasz, A. Schubert, T. Vicsek, Physica A 311, 590 (2002).

[13] I. Farkas, I. Derényi, H. Jeong, Z. Néda, Z.N. Oltvai, E. Ravasz, A. Schubert, A.-L. Barabási, T. Vicsek, Physica A 314, 25 (2002).

[14] K.E. Lee, J.W. Lee, B.H. Hong, Comput. Phys. Commun. 177, 186 (2007).

[15] H.-J. Kim, Y. Lee, B. Kahng, I. Kim, J. Phys. Soc. Jpn. 71, 2133 (2002).

[16] G. Caldarelli, Scale-Free Networks, Oxford University Press, Oxford 2013.

[17] A.-L. Barabasi, R. Albert, Science 286, 509 (1999).

[18] F. Liljeros, C.R. Edling, Y. Ĺberg, L.A. Nunes Amaral, H.E. Stanley, Nature 411, 907 (2001).

[19] Y.U. Saito, T. Watanabe, M. Iwamura, Physica A 383, 158 (2007).

[20] W. Souma, Y. Fujiwara, H. Aoyama, Physica A 324, 369 (2003).

[21] T. Petermann, P. De Los Rios, Europ. Phys. J. B 38, 201 (2004). 
[22] Y. Virkar, A. Clauset, Ann. Appl. Statist. 8, 89 (2014).

[23] R. Cohen, S. Havlin, Phys. Rev. Lett. 90, 058701 (2003).

[24] Y.-Z. Yan, W.-Q. Tu, L.-J. Tang, J. Appl. Math. Comput. 43, 91 (2013).

[25] C. Cooper, A. Frieze, Random Struct. Algor. 22, 311 (2003).

[26] G. Caldarelli, M. Catanzaro, Physica A 338, 98 (2004).

[27] R. Grassi, Physica A 389, 2455 (2010).
[28] S. Battiston, M. Catanzaro, Europ. Phys. J. B 38, 345 (2004).

[29] M.L. Goldstein, S.A. Morris, G.G. Yen, Europ. Phys. J. B 41, 255 (2004).

[30] M. Brzezinski, Physica A 406, 155 (2014).

[31] A. Clauset, C.R. Shalizi, M.E.J. Newman, SIAM Rev. 51, 661 (2009).

[32] P. Erdös, A. Renyi, Publ. Math. 6, 290 (1959). 